Communications in Physics, Vol. 24, No. 3S2 (2014), pp. 121-129

DOI:10.15625/0868-3166/24/3S2/5057

\title{
INFLUENCE OF SURFACE PLASMON RESONANCE ON FLUORESCENCE EMISSION OF DYE-DOPED NANOPARTICLES
}

\author{
CHU VIET HA \\ College of Education, Thai Nguyen University \\ and \\ Institute of Physic, Vietnam Academy of Science and Technology \\ J. C. BROCHON \\ Laboratoire de Biotechnologie et Pharmacologie Appliquee, ENS Cachan, France \\ TRAN HONG NHUNG \\ Institute of Physic, Vietnam Academy of Science and Technology \\ E-mail: thnhung@iop.vast.ac.vn
}

Received 20 June 2014

Accepted for publication 20 August 2014

\begin{abstract}
The influence of the surface plasmon of gold nanoparticles on the optical properties of the fluorescent nanoparticles in aqueous solution have been investigated. The fluorescence of nanoparticles can be enhanced or quenched in the presence of gold nanoparticles depending on the domination of energy transfer mechanisms: radiating surface plasmon coupling emission or Förster energy transfer from fluorescent particles to gold particles, which exciting absorbing plasmon. The fluorescence enhancement or quenching is attributed to the increase or decrease of radiative recombination rates, respectively. The parameters of the energy transfer between fluorescent nanoparticles (dye molecules encapsulated in silica nanoparticles) and nano golds have been estimated. The results show that the interactions between nanoparticles depend on the size of both fluorophores (as donors) and gold nanoparticles (as acceptors).
\end{abstract}

Keywords: dye-poped nanoparticles, surface plasmon resonance, fluorescence.

\section{INTRODUCTION}

Surface plasmons are the coupled modes of electromagnetic field and free electrons in metal. The interaction of fluorescent molecules with plasmons in metallic nanostructures and the physical phenomena related to nanoscale confinement of light have attracting the interest of physicists over the past years $[1,2]$. Recently, there are a numerous studies of metal-enhanced fluorescence by metal nanoparticles (NPs) due to the interactions between fluorophores and the surface plasmon field of the metal NPs [3-8]. The fluorescence of fluorophore nearby a metal can be enhanced or quenched depending on the distance between the fluorophores and metal NPs. When a fluorophore is placed at a suitable distance from a metallic NP or surface, their emission decay rates change, result in the changes of lifetime and quantum yield $[3,4,8]$. The interaction 
between fluorophores and metallic NPs depends not only on the distance between them but also on the overlap of the plasmon absorption spectra of the metal and the emission spectra of the fluorophores [9], and the relative orientation of the dipole momentum of fluorophore and metal surface $[10,11]$. The radiating plasmons model is used when a particular metal structure will enhance fluorescence [5]. Currently, the fluorophores in interactions are implied dye molecules and quantum dots. Because of their small dimention, the size-effects have never been paid attention. But if a fluorescent NP could be placed near by a metal instead of the dye molecule, the parameters of the interactions will be changed. The optimal fluorescence enhancement has been observed by mixing directly the fluorescent and gold NPs and reported in Ref. [16].

In the present work, the radiative and nonradiative recombination rates have been investigated in the case of fluorescence enhancement and quenching. The fluorescence enhancement factors also have been estimated for NPs with different size. The results were compared with those of free dye molecules

\section{MATERIALS AND METHODS}

The fluorescent NPs used in this work are the orange fluorescent $100 \mathrm{~nm}$ carboxylatemodifed FluoSpheres $囚$ beads (Invitrogen), which have absorption peak at $540 \mathrm{~nm}$ and emission peak at $560 \mathrm{~nm}$, called the orange beads (OBs). Each bead contains $\sim 3000$ Cyanine3 (Cy3) dye molecules. The aqueous solution of OBs is prepared with the concentration of $3.64 \times 10^{6}(\mathrm{~mL})^{-1}$. The optical characterization of the OBs solution was investigated by adding the $20 \mathrm{~nm}$ colloidal gold nanoparticles (GNPs), which were purchased from BBinternational with optical density of 1. The quantities of GNPs added into $1.5 \mathrm{~mL}$ OBs solution were varied from 1 to $250 \mu \mathrm{L}$. The GNPs and OBs have citrate and carboxyl groups on their surface, respectly. So they cannot aggregate to each other in mixture solution.

UV-Vis spectra of the OBs-GNPs mixtures have been recorded by spectrophotometer Jasco V-600. The photoluminescence spectra of these mixtures were performes on fluorometer Cary Eclipse (Varian) at room temperature. The fluorescence lifetime was detected under two photon excitation of Ti: Sapphire laser (1000 nm, $80 \mathrm{fs)} \mathrm{MaiTai,} \mathrm{Spectra} \mathrm{Physics.} \mathrm{A} \mathrm{time-correlated}$ single-photon counting SPC-430 card (Becker-Hickl GmbH) was used for the acquisition. The fluorescence quantum yield of OBs in the mixture has been estimated. In order to compare the influence of surface plasmon field of GNPs on optical properties of OBs. The optical characteristics of $\mathrm{Cy} 3$ free dye - GNPs mixtures were investigated in order to compare with those of OBs-GNPs mixtures

\section{RESULTS AND DISCUSSIONS}

Fig. 1 presents the transmission electron microscopy (TEM) image (JEOL JEM 1011) of the OBs. TEM image of GNPs is shown in Fig. 2. Fig. 3 presents the normalized of absorption and fluorescence spectra of OBs together with plasmon resonance spectra of $20 \mathrm{~nm}$ GNPs. The optical spectra of Cy3 dye are shown in Fig. 4.

III.1. Investigating of recombination rates of the OBs in the case of fluorescence enhancement or quenching with the presence of GNP in solution

The measurement of absorption spectra in UV-Vis region of the aqueous solution of the mixture OBs and GNPs has been performed. The UV absorption spectra of the mixture OBs and 


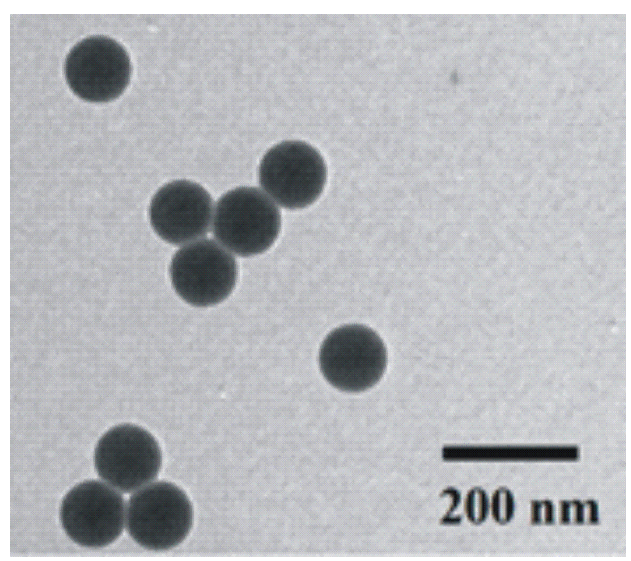

Fig. 1. TEM image of OBs

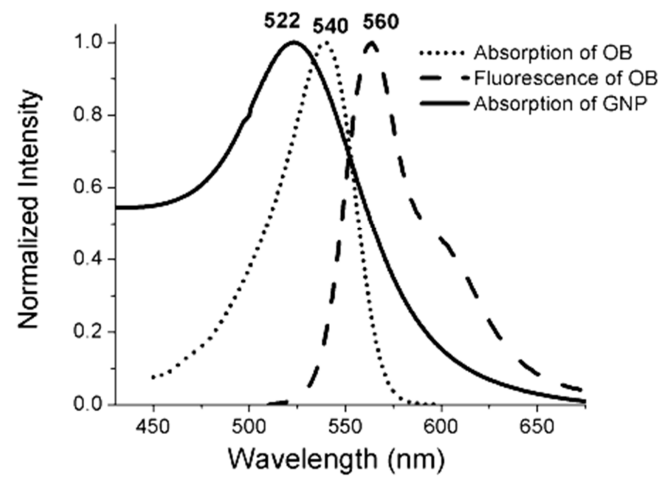

Fig. 3. Spectra of OBs and GNPs

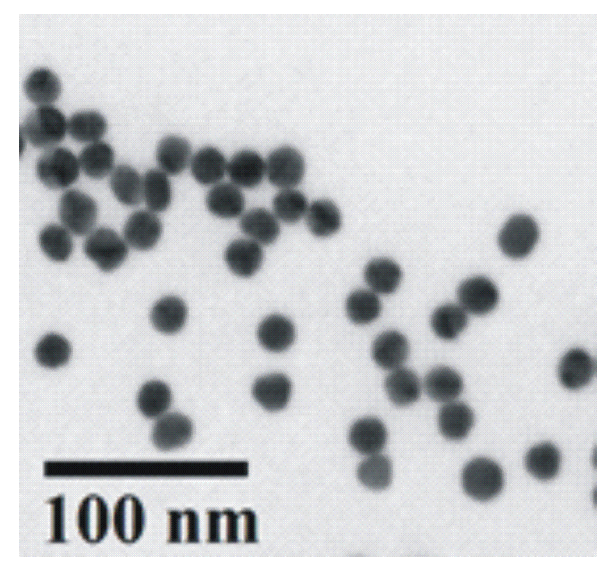

Fig. 2. TEM image of GNPs

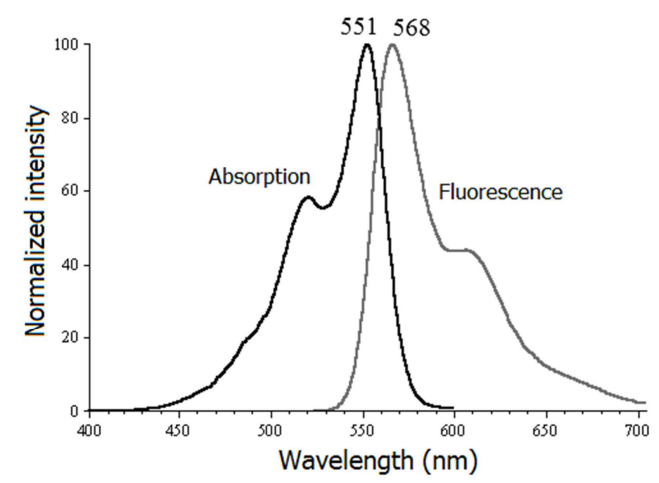

Fig. 4. Spectra of $\mathrm{Cy} 3$ dye in solution

GNPs at different concentration of GNPs are shown in Fig. 5. The absorbance increases with the increase of GNPs quantity in solution. There is no peak of plasmon absorption of GNP at 522 $\mathrm{nm}$ is observed, just only peak at $540 \mathrm{~nm}$ is observed. The shape of absorption spectra of OBs with various GNP's concentrations appeared to be unchanged. The result can be explained by the absorbance of the GNPs smaller than the absorbance of OBs. The increase of absorbance shows the contribution of GNPs in the mixture.

The fluorescence spectra of the mixture of OBs and GNPs are measured under excitation of $532 \mathrm{~nm}$. There is optimal enhancement of the fluorescence emission of the mixture compared to OBs solution in the absence of GNPs. The fluorescence intensity increases when the GNP quantity increase from 0 to $210 \mu \mathrm{L}$ (Fig. 6a) and then decreases when the GNP quantity continues increases (Fig. 6b). Both spectra exhibit a fluorescence peak at around $560 \mathrm{~nm}$. The spectra shape is mostly unchanged in the presence of the GNPs. The strongest fluorescence enhancement factor is 4.5 corresponding to $210 \mu \mathrm{L}$ GNP added. The dependence on the GNP concentration in solution of the fluorescence intensity is described in Fig. 7. The fluorescence enhancement can be explained by interaction between OBs and GNPs [12-15]. A numerous reports have shown that the interaction between fluorophores and metal nanoparticles depending on the distance between 


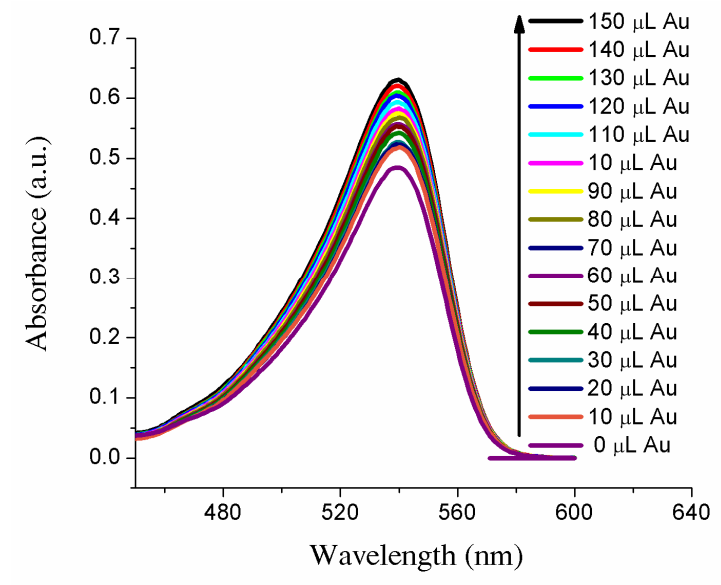

Fig. 5. Absorption spectra of the mixture OBs and GNPs at different concentration of GNPs

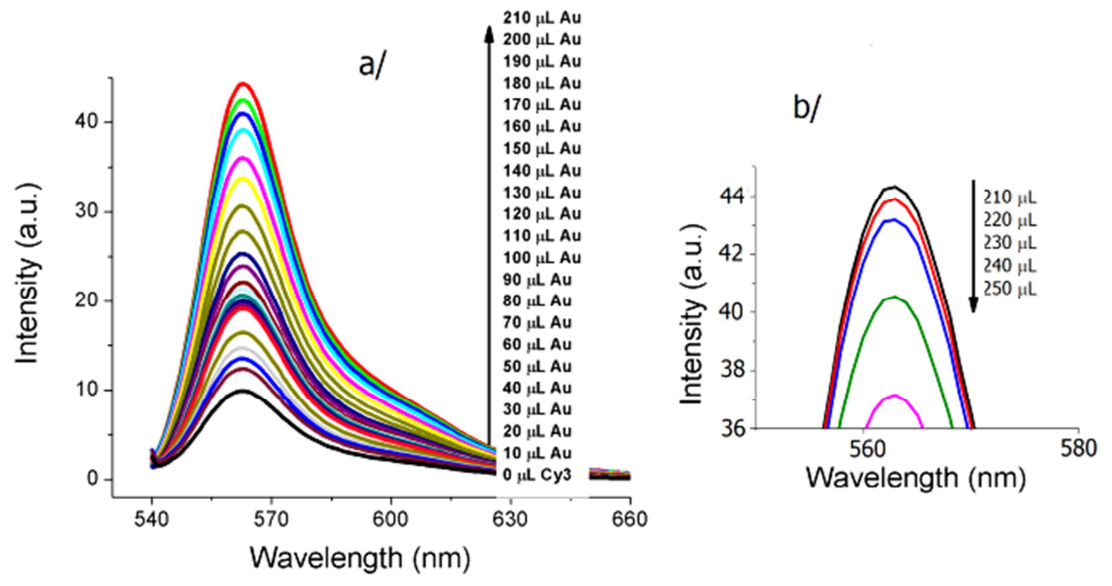

Fig. 6. Fluorescence spectra of the mixture OBs and GNPs at different concentration of GNPs under excitation of $532 \mathrm{~nm}$ under room temperature

them. Nearby conducting metallic nanoparticles can alter the free space conditions that increase or decrease the incident electric field due to increasing or decreasing the radiative decay rate. This can be explained by the changes in photonic mode density, with large photonic density influencing the radiative decay rates and increasing the number of pathways available to release its excited energy $[3,6]$. We can realize that when a fluorophore placed nearby a metallic nanostructure, two interaction mechanisms exist parallel to each other: the radiating surface plasmon coupling emission of fluorophore makes the fluorescence enhance $[3,5]$ and the energy transfer from the fluorophore to the metal exciting absorbing plasmon makes the fluorescence quench. The two mechanisms are always in situation of competition, one of them is dominant will make the fluorescence enhance or quench. The fluorescence quenching of fluorophore can be explained like the Förster energy transfer between two fuorophores that makes the fluorescence of the donor decrease. This energy 
transfer can be attributed to the surface energy transfer mechanism (SET) [17-20]. To investigate the recombination rates in the cases of enhancement and quenching fluorescence, the lifetime and quantum yield of the OBs solution with the presence of GNPs have been measured. The quantum yield of the mixture of OBs and GNPs has been estimated via comparing with the quantum yield Rhodamine 6G (R6G) dye in aquoues solution at the same absorbance. The quantum yield of R6G dissolved in water with the excitation of $480 \mathrm{~nm}$ is 0.95 [21]. By the normalization of absorption spectra, we can estimate the quantum yield of the OBs and GNPs mixture solution.

The lifetime of OBs with the appearance of GNPs has been calculated from the fluorescence decay measured by two-photon excitation using a Ti-Sapphire laser at room temperature. The lifetime $\tau$ of a fluorophore related to the quantum yield $Q$ by a simple formula [22]:

$$
Q=\frac{\Gamma_{r}}{\Gamma_{r}+\Gamma_{n r}}=\frac{\Gamma_{r}}{\Gamma_{t o t}}=\Gamma_{r} \cdot \tau
$$

where $\Gamma_{r}$ is radiative recombination rate, $\Gamma_{n}$ is nonradiative rate including all possible nonradiative decay processes. When the interactions between the fluorophore and the GNPs appear, it could change the recombination rate that causes the changes of the quantum yield and the lifetime of the flourophore. By calculating the lifetime and measuring quantum yield, the recombination rates of OBs with different GNP concentrations can be calculated completely. The results are shown in Table 1.

This is result of the increase of radiative recombination rate and decrease of nonradiative recombination rate. The fluorescence enhancement factor and recombination rates are described in Fig. 8. The enhancement factor is determined by the ratio of fluorescence intensity of the OBs in the presence and absence of GNPs. From Fig. 8 we can see that the increase of fluorescence intensity is due to the increase of radiative recombination rate and decrease of nonradiative recombination rate. When the fluorescence intensity decreases, the radiative recombination rate decreases and the non radiative recombination rate increases. The strongest enhancement is corresponding to the highest radiative recombination rate and the smallest non radiative recombination rate.

Table 1. The lifetime and recombination rates of OBs with different GNP quantities

\begin{tabular}{cccccc}
\hline \hline $\begin{array}{c}\text { The GNP } \\
\text { quantity in the } \\
\text { mixture }(\mu \mathbf{L})\end{array}$ & $\begin{array}{c}\text { Lifetime } \\
(\mathbf{n s})\end{array}$ & $\begin{array}{c}\text { Quantum } \\
\text { yield }\end{array}$ & $\begin{array}{c}\text { Radiative } \\
\text { recombination } \\
\text { rate }\left(\mathbf{s}^{-1}\right)\end{array}$ & $\begin{array}{c}\text { Radiative } \\
\text { recombination } \\
\text { rate }\left(\mathbf{s}^{-1}\right)\end{array}$ & $\begin{array}{c}\text { Total } \\
\text { recombination } \\
\text { rate }\left(\mathbf{s}^{-1}\right)\end{array}$ \\
\hline \hline 0 & 4.56 & 0.11 & $2.5 \times 10^{7}$ & $19.4 \times 10^{7}$ & $21.9 \times 10^{7}$ \\
\hline 20 & 4.32 & 0.22 & $5 \times 10^{7}$ & $18.2 \times 10^{7}$ & $23.2 \times 10^{7}$ \\
\hline 70 & 4.19 & 0.31 & $7.3 \times 10^{7}$ & $16.4 \times 10^{7}$ & $23.7 \times 10^{7}$ \\
\hline 120 & 4.44 & 0.39 & $8.7 \times 10^{7}$ & $13.8 \times 10^{7}$ & $22.5 \times 10^{7}$ \\
\hline 150 & 4.49 & 0.50 & $1.1 \times 10^{8}$ & $11.2 \times 10^{7}$ & $22.2 \times 10^{7}$ \\
\hline 210 & 4.55 & 0.70 & $1.5 \times 10^{8}$ & $6.9 \times 10^{7}$ & $21.9 \times 10^{7}$ \\
\hline 220 & 4.28 & 0.70 & $1.6 \times 10^{8}$ & $7.4 \times 10^{7}$ & $23.4 \times 10^{7}$ \\
\hline 250 & 4.31 & 0.58 & $1.3 \times 10^{8}$ & $10.2 \times 10^{7}$ & $23.2 \times 10^{7}$ \\
\hline \hline
\end{tabular}


Table 1 show that the fluorescence enhancement is corresponding to the enhancement of quantum.

The fluorescence quenching of the OBs with the presence of GNPs is attributed to the energy transfer from OB to GNP causing the localized absorption plasmons. Consider the OB is donor GNP is acceptor, the energy transfer efficiency $(E)$ and energy transfer rate $(k)$ related to the lifetime of OBs without GNPs $\left(\tau_{0}\right)$ and the lifetime of OBs with GNPs $\left(\tau_{D}\right)$ by formulas [22]:

$$
\begin{aligned}
& E=1-\frac{\tau_{D}}{\tau_{0}} \\
& k=\frac{1}{\tau_{D}}-\frac{1}{\tau_{0}}
\end{aligned}
$$

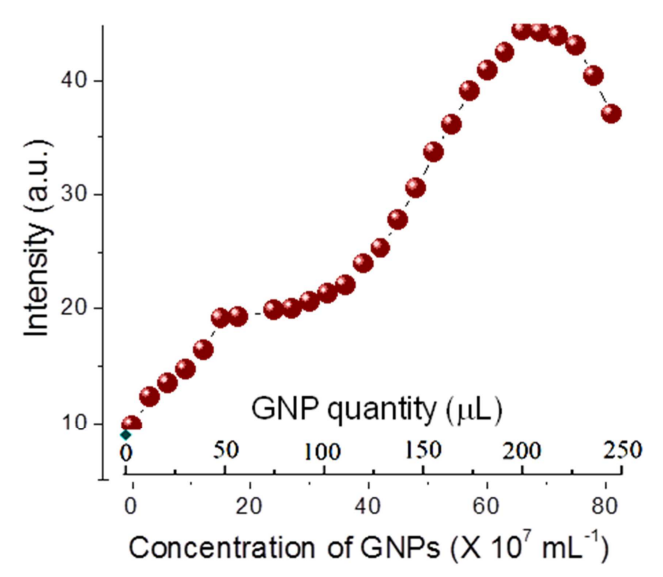

Fig. 7. Fluorescence intensity of OBs versus the concentration of $20 \mathrm{~nm}$ GNPs

Table 2. The energy transfer rate and the energy transfer efficiency from OB to GNP with different GNP quantities

\begin{tabular}{cccc}
\hline \hline $\begin{array}{c}\text { The GNP quantity } \\
\text { in the mixture }(\boldsymbol{\mu} \mathbf{L})\end{array}$ & $\begin{array}{c}\text { Lifetime } \\
(\mathbf{n s})\end{array}$ & $\begin{array}{c}\text { The energy } \\
\text { transfer rate }\left(\mathbf{s}^{-1}\right)\end{array}$ & $\begin{array}{c}\text { The energy } \\
\text { transfer efficiency }(\boldsymbol{\%})\end{array}$ \\
\hline \hline 0 & 4,56 & - & - \\
\hline 20 & 4,32 & $0,1 \times 10^{8}$ & 5,2 \\
\hline 70 & 4,19 & $0,2 \times 10^{8}$ & 8,1 \\
\hline 120 & 4,44 & $0,06 \times 10^{8}$ & 2,6 \\
\hline 150 & 4,49 & $0,03 \times 10^{8}$ & 1,5 \\
\hline 210 & 4,55 & $0,005 \times 10^{8}$ & 0,2 \\
\hline 220 & 4,28 & $0,14 \times 10^{8}$ & 6,1 \\
\hline 250 & 4,31 & $0,13 \times 10^{8}$ & 5,5 \\
\hline \hline
\end{tabular}

Table 2 presents the results about the energy transfer efficiency and energy transfer rate with different GNP quantities. The Förster energy transfer efficiency and the quantum yield of 


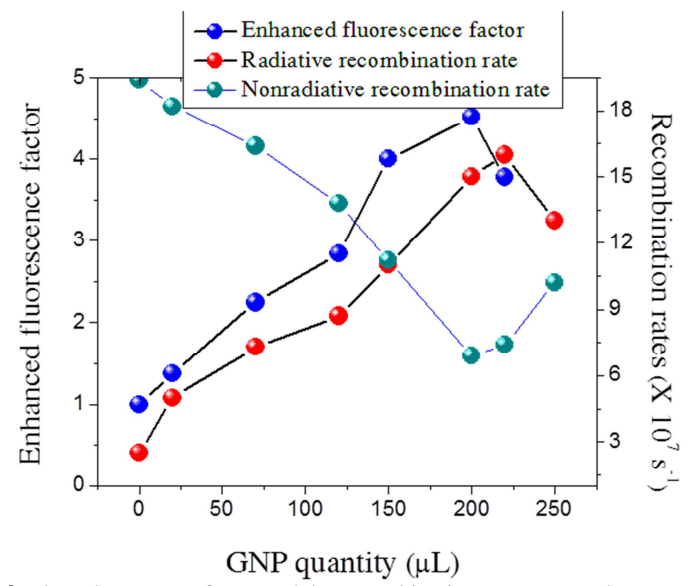

Fig. 8. The enhancement factor and the recombination rates versus the concentration of $20 \mathrm{~nm}$ GNPs

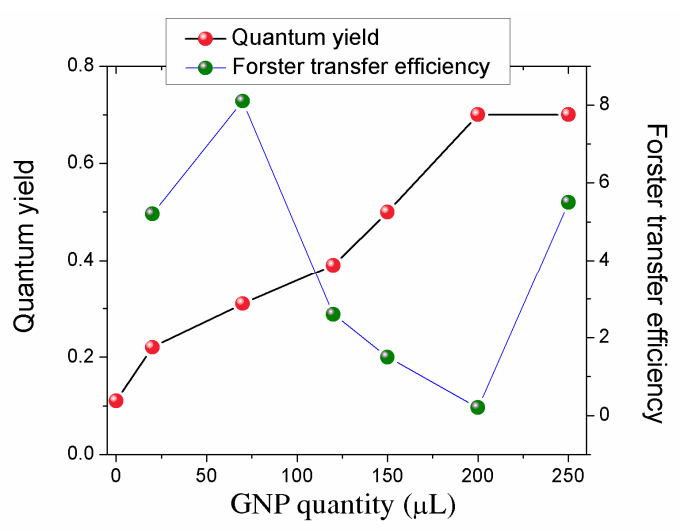

Fig. 9. The Förster energy transfer efficiency compared with the quantum yield versus the concentration of $20 \mathrm{~nm}$ GNPs

OBs versus the quantity of GNPs are described on Fig. 9. We can see that the quantum yield tends to increase or decrease in contrast to the trend of Förster energy transfer efficiency. Förster energy transfer efficiency decreases due to the decrease of nonradiative recombination rate and the increase of nonradiative recombination rate.

\section{III.2. Photoluminescence properties of $\mathrm{Cy} 3$ dye in surface plasmon resonance effects of GNPs}

The free Cy3 dye has been dissolved in aqueous solution with the same absorbance of the OBs solution above. The quantum yield of $\mathrm{Cy} 3$ dye solution with the absence of GNPs is estimate by $0.1 \%$. The investigation results show that the enhancement of the fluorescence is due to the increase of radiative recombination rate and decrease of non radiative recombination rate; the quenching of the fluorescence is due to the decrease of radiative recombination rate and increase of non radiative recombination rate. We have compared the fluorescence enhancement between the OBs (Cy3 dye doped nanoparticles) and free Cye dye in solution with the appearance of GNPs.

Fig. 10 presents the dependence on the GNP concentration in solution of the fluorescence intensity of $\mathrm{OBs}$ and free $\mathrm{Cy} 3$. The maximum enhancement factor of $\mathrm{Cy} 3$ dye fluorescence is $\sim 2$ while the maximum enhancement factor of OBs rises up to 4.5 times. We can see that the energy transfers between fluorescent NPs and gold nanoparticles have the superior enhancement factors than that between dye and gold nanoparticles. The brightness and fluorescence signal of one OB is much higher than a free dye molecule due to the number of dye molecule in one OB is very large ( $\sim 3000$ molecules per bead). This leads to the fluorescence enhancement factors depend on the nanoparticle size or the number of dye molecules in a nanoparticle. The number of dye molecules is larger, the enhancement factors are higher. This implies that the resonant interactions between dye molecules in one nanoparticle and these interactions in turn can act in resonance with surface plasmon, leading to the improvement of fluorescence enhancement factors. This plays an important role to control the fluorescence by metal nanoparticles. 


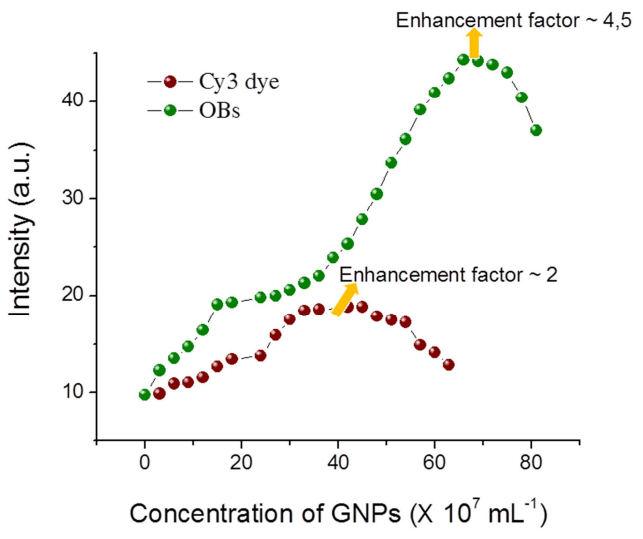

Fig. 10. Fluorescence intensity of $\mathrm{Cy} 3$ dye compared to OBs versus the concentration of $20 \mathrm{~nm}$ GNPs

The interaction between the fluorescence nanoparticles and nano gold with the different size of nanoparticles will be studied more detail and present in next reports. We focus on studying optical properties of dye doped silica nanoparticles with different size in interaction with gold nanoparticles. We also study effects of the overlap between the emission spectra of fluorescence nanoparticle and plasmon resonance absorption spectra of gold nanoparticles on fluorescence enhancement and quenching.

The effects of the surface plasmon of gold nanoparticles on the optical properties of the fluorescent nanoparticles have been investigated. The gold nanoparticles can enhance or quench the fluorescence of fluorescent nanoparticles such as dye-doped silica, polystyrene nanoparticles, and quantum dots depending on energy transfer mechanisms, the domination of radiating surface plasmon coupling emission or the Förster energy transfer from fluorescent particles to gold particles exciting absorbing plasmon. The fluorescence enhancement and quenching are attributed to the increase and decrease of radiative recombination rates, respectively.

The parameters of the energy transfer between fluorescent nanoparticles and nano golds have been estimated. The results show that the interactions between nanoparticles depend on the size of both fluorophores (as donors) and gold nanoparticles (as acceptors). A model "antenna" is given to explain the particle-size dependence of the critical distance Förster energy transfer between nanoparticles.

\section{CONCLUSION}

The effects of the surface plasmon of gold nanoparticles on the optical properties of the fluorescent nanoparticles have been investigated. The fluorescence enhancement and quenching are attributed to the increase and decrease of radiative recombination rates, respectively. The gold nanoparticles can enhance or quench fluorescence depending on energy transfer mechanisms: when the radiating surface plasmon coupling emission process dominates, the fluorescence of the fluorophore nanoparticles will be enhanced due to the increase of radiative recombination rate. When the Förster energy transfer from fluorescent particles to gold particles exciting absorbing 
plasmon dominates, the fluorescence intensity of particles will be decreased due to the decrease of radiative recombination rates, resulting the fluorescence quenching.

The fluorescence enhancement factors depend on the nanoparticle size or the number of dye molecules in a nanoparticle. The number of dye molecules is larger, the enhancement factors are higher.

\section{ACKNOWLEDGMENTS}

This work was supported by National Project No. 01/2/2011/HD-NCCBUD.

\section{REFERENCES}

[1] W. L. Barnes, A. Dereux, and T. W. Ebbesen, Nature 424 (2003) 824-830

[2] S. Maier, "Plasmonics: Fundamentals and Applications"; Springer Science+Business Media LLC: New York, NY, USA, 2004

[3] C. D. Geddes and J. R. Lakowicz, Journal of Fluorescence, 12(2) (2002) 121 - 128

[4] K. Aslan, I. Gryczynski, J. Malicka, E. Matveeva, J.R. Lakowicz, C.D. Geddes, ”Metal-Enhanced Fluorescence: An Emerging Trend in Biotechnology", Current Opinion in Biotechnology, Vol. 16, No.1, 2005, pp. 55-62.

[5] J. R. Lakowicz, Analytical Biochemistry 3372005 171-194

[6] K. Aslan, J. Huang, G. M. Wilson, and C. D. Geddes, Journal of the American Chemical Society 128 (2006) 4206-4207.

[7] Vitaliy N. Pustovit, Physica B 405 (2010) 754-757

[8] Y. Fu, J. Zhang, and J. R. Lakowicz, Plasmon-Controlled Fluorescence: Applications to Sensing and Single Molecule Detection, Proceeding of SPIE 7569756903 (2010) 6 pp.

[9] M. Olejnik, . Bujak and S. Mackowski, International Journal of Molecular Sciences 13 (2012) 1018-1028

[10] R. Chance, A. Prock, and R. Silbey, Advances in Chemical Physics 37 (1978) 1-65

[11] P. M. Whitmore, H. J. Robota, C. B. Harris, Journal of Chemical Physics 77 (1982) 1560-1569.

[12] Krishanu Ray, Ramachandram Badugu, and Joseph R. Lakowicz, J. Am. Chem. Soc. 128 (2006) 8998-8999

[13] Dae-Ryong Jung, Jongmin Kim, Seunghoon Nam, Changwoo Nahm, Hongsik Choi, Jae Ik Kim, Junhee Lee, Chohui Kim, and Byungwoo Park, Appl. Phys. Lett. 99 (2011) 041906

[14] C. S. S. R. Kumar, Biofunctionalization of Nanomaterials Nanotechnologies for the Life Sciences 1, WILEYVCH Verlag GmbH \& Co. KGaA, Weinheim, ISBN: 3-527-31381-8, 2005.

[15] J. Yan, M. C. Estévez, J. E. Smith, K. Wang, X. He, L. Wang, and W. Tan, Nanotoday 2(3) (2007) 44-50

[16] V.H. Chu, E. Fort, T.H.L Nghiem and H.N. Tran, Advances in Natural Sciences: Nanoscience and Nanotechnology 2 (2011) 045010

[17] T. Sen, S. Sadhu, and A. Patra, Appl Phys Lett 91 (2007) 043104

[18] M. P. Singh and G. F. Strouse, J. Am. Chem. Soc. 132(27) (2010) 9383 - 9391

[19] C. S. Yun, A. Javier,T. Jennings, M. Fisher, S. Hira, S. Peterson, B. Hopkins, N.O. Reich, and G. F. Strouse (2005), "Nanometal Surface Energy Transfer in Optical Rulers, Breaking the FRET Barrier", J. Am. Chem. Soc. 127(9), 3115-3119

[20] T. L. Jennings, M. P. Singh, and G. F. Strouse, J. Am. Chem. Soc. 128 (2006) 5462 - 5467

[21] R. F. Kubin and A. N. Fletcher, J. Luminescence 27 (1982) 455-462.

[22] J. R. Lakowicz, Principl of Fluorescence Spectroscopy, Springer, 1999. 